\title{
Face adaptation effects show strong and long-lasting transfer from lab to more ecological contexts
}

\author{
Claus-Christian Carbon ${ }^{1}$ and Thomas Ditye ${ }^{2}$ \\ ' Department of General Psychology and Methodology, University of Bamberg, Bamberg, Germany \\ ${ }^{2}$ Institute of Cognitive Neuroscience, University College London, London, UK
}

Edited by:

Peter James Hills, Anglia Ruskin

University, UK

Reviewed by:

Karla K. Evans, Harvard Medical School, USA

Anthony Little, University of Stirling, UK

\section{*Correspondence:}

Claus-Christian Carbon, Department of General Psychology, University of Bamberg, Markusplatz 3, D-96047

Bamberg, Germany.

e-mail:ccc@experimental-

psychology.com
A review on recent experiments on figural face aftereffects reveals that adaptation effects in famous faces can last for hours up to days. Such adaptations seem to be highly reliable regarding test-retest designs as well as regarding the generalizability of adaptation across different adaptation routines and adaptations toward different kinds of facial properties. However, in the studies conducted so far, adaptation and the subsequent test phase were carried out in typical laboratory environments. Under these circumstances, it cannot be ruled out that the observed effects are, in fact, episodic learn-test compatibility effects. To test for ecological validity in adaptation effects we used an adaptation paradigm including environmental and social properties that differed between adaptation and test phase. With matched samples $(n 1=n 2=54)$ we found no main effects of experimental setting compatibility resulting from varying where the tests where conducted (environmental condition) nor any interaction with effects of stimulus compatibility resulting from varying stimulus similarity between adaptation and test phase using the same picture, different pictures of the same person, or different persons (transfer). This indicates that these adaptation effects are not artificial or merely lab-biased effects. Adaptation to face stimuli may document representational adaptations and tuning mechanisms that integrate new visual input in a very fast, reliable, and sustainable way.

Keywords: face adaptation, ecological testing, external validity, face representation, figural aftereffects, face veridicality aftereffect, familiar faces, plasticity

\section{INTRODUCTION}

Adaptation is a continuous process that enables us to tune our cognitive apparatus to new, changing, and dynamic aspects of our external world. It takes effect across sense modalities in lower as well as higher areas of sensory processing by changing the firing patterns in neural populations that are sensitive to a given adaptive stimulus. In visual perception, for instance, exposure to color or motion direction shapes the perception of these features in subsequent neutral (i.e., achromatic or stationary, respectively) stimuli in a way that corresponds to a shift in baseline induced by adaptation.

Experimental research in recent years tells us that adaptation effects in the domain of faces are reliable (Rhodes et al., 2003), show robustness against many experimental variables such as size, orientation, and affine distortions (for an overview see Webster and Macleod, 2011) and are also sustainable (Carbon and Ditye, 2011). Carbon and colleagues have shown that adaptation toward distorted familiar faces can last up to $80 \mathrm{~min}$ (Carbon and Leder, 2006), several days (Carbon et al., 2007), or even 1 week (Carbon and Ditye, 2011), indicating instant and robust changes of the cognitive representation toward newly incoming visual information. In these studies, participants adapted to configurally distorted faces and were later asked to pick the veridical versions of these faces from of a sequence of different versions with varying distortions. After adaptation, the perceived veridical version was shifted toward the adapting distortion.
These long-term adaptation paradigms included an adaptation block, during which a number of manipulated faces were shown for multiple trials, followed by a delay period of hours or even days, and a subsequent test session. In contrast, in most studies looking at immediate (i.e., short-term) adaptation effects, the adaptation face was immediately followed by a test face, thus measuring aftereffects on a trial-by-trial basis rather than blockby-block. The question has been raised if long-term adaptation effects are the result of neural mechanisms that are similar to those of short-term adaptation as some of the reported effects could be also explained by generic effects of episodic memory adjustments. Perception might be temporarily biased as a specific test situation - the laboratory - provides reliable cues for episodic memory. Tulving defined episodic memory as a memory system that "receives and stores information about temporally dated episodes or events, and temporal-spatial relations among these events... [which] is always stored in terms of its autobiographical reference..." (Tulving, 1972, p. 385). On the basis of this original definition we could assume that the typical setting of face adaptation studies conducted under strict experimental conditions in laboratories (see Table 1 for a list of example laboratory based face adaptation studies) with reliable environmental, situational, and social settings induce strong episodic memory traces during the adaptation phase that might be reactivated when the participants are tested for supposed adaptation effects. 
If adaptation effects are primarily based on such episodic effects, then adaptation effects should decrease or be absent when environmental, situational, and social factors vary or change between adaptation and test phase.

\section{THE PRESENT STUDY}

In the present study, we tested the ecological validity of sustained face adaptation effects. Therefore, the environmental, situational, and social factors were set very differently in the adaptation phase and the test phase (environmental condition). The phases were separated by an extensive delay of at least 7 days. Test included the same pictures that were used for adaptation (picture) plus different pictures of the same persons (identity), and pictures of completely

Table 1 | Lab-based studies on face adaptation.

\begin{tabular}{|c|c|c|}
\hline Study & Transfer & Delay \\
\hline Webster and Maclin (1999) & None & $\mathrm{n} / \mathrm{a}$ \\
\hline Leopold et al. (2001) & Anti-faces, position, size & $\begin{array}{l}150,300 \\
600,1200 \\
2400 \mathrm{~ms}\end{array}$ \\
\hline Rhodes et al. (2003) & Orientation & $500 \mathrm{~ms}$ \\
\hline Webster et al. (2004) & $\begin{array}{l}\text { Gender, ethnicity, } \\
\text { expression }\end{array}$ & $250 \mathrm{~ms}$ \\
\hline Carbon and Leder (2005) & None & $4 \mathrm{~s}, 5 \mathrm{~min}$ \\
\hline Kovacs et al. (2005) & Retinal position & $500 \mathrm{~ms}$ \\
\hline Little et al. (2005) & Different faces; gender & $\mathrm{n} / \mathrm{a}$ \\
\hline Leopold et al. (2005) & Anti-faces & $\mathrm{n} / \mathrm{a}$ \\
\hline Carbon and Leder (2006) & None & $80 \mathrm{~min}$ \\
\hline Carbon et al. (2007) & $\begin{array}{l}\text { Different pictures/ } \\
\text { different persons }\end{array}$ & $5 \mathrm{~min}, 24 \mathrm{~h}$ \\
\hline Fang et al. (2007) & $\begin{array}{l}\text { Different faces; inverted } \\
\text { faces }\end{array}$ & $1 \mathrm{~s}$ \\
\hline Rhodes et al. (2007) & Anti-faces, size & $1000 \mathrm{~ms}$ \\
\hline Kloth and Schweinberger (2008) & Size & $7 \mathrm{~min}$ \\
\hline Barrett and O'Toole (2009) & Age-groups & $100 \mathrm{~ms}$ \\
\hline Hills et al. (2010) & Imagery/perception & $5 s$ \\
\hline Carbon and Ditye (2011) & $\begin{array}{l}\text { Different pictures/ } \\
\text { different persons }\end{array}$ & $24 \mathrm{~h}, 7$ days \\
\hline Hole (2011) & Upside-down; stretched & $\leq 2 \min$ \\
\hline
\end{tabular}

Overview of a selection of lab-based empirical studies on face adaptation in chronological order systematizing the transfer conditions between adaptation and test ("transfer") and the documented duration of the adaptation effects. new faces (novel). The test for such transfer effects can add relevant information about the hierarchal locus of adaptation effects in face perception. While transfer of distortion effects across identities have been shown before (Webster and Maclin, 1999; Carbon et al., 2007; Carbon and Ditye, 2011), face identity aftereffects have shown to be highly dependent on viewpoint (Benton et al., 2006).

\section{MATERIALS AND METHODS PARTICIPANTS}

One hundred and eight undergraduate students (92 female; mean age: 22.6 years, range: $18-43)$ took part on a volunteer basis. Participants were naïve to the purpose of the experiment and had normal or corrected-to-normal vision, as assessed by the SnellenEye chart vision test. Participants were assigned to one of two levels of the environmental condition factor: half of the participants (43 female; mean age: 22.6 years, range: $18-43$ ) to the formal experimental laboratory setting (same), the other half ( 49 female; mean age: 22.6 years, range: $18-37$ ) to the informal leisure room setting (different). Groups were matched according to participants' age.

\section{APPARATUS AND STIMULI}

We used two different high-quality photo-portraits (images A and B) of 27 celebrities (frontal view; $220 \times 240$ pixels) who were well known to the participants (pop stars, politicians, super models, sportsmen: Pamela Anderson, Franz Beckenbauer, Boris Becker, Pierce Brosnan, George W. Bush, Nicholas Cage, Bill Clinton, George Clooney, Cindy Crawford, Tom Cruise, Princess Diana, Cameron Diaz, Verona Feldbusch, Thomas Gottschalk, Oliver Kahn, Nicole Kidman, Helmut Kohl, Madonna, Angela Merkel, Jack Nicholson, Brad Pitt, Julia Roberts, Claudia Schiffer, Gerhard Schröder, Michael Schumacher, Arnold Schwarzenegger, Rudi Völler). For the adaptation phase we attempted to create effective adaptors to achieve a strong adaptation effect. Following the advice of Carbon et al. (2007), we decided to create different versions of the original portraits by generating strong configural distortions: We gradually compressed or extended the distances between the eyes and the mouth for each picture by an amount of approximately 20 pixels (Figure 1). Each celebrity was randomly allocated to one of three stimulus sets (consisting of nine celebrities) that corresponded to the three levels of the factor transfer: picture, identity, and novel. The experiment was controlled by the experimental software PsyScope X B.46 (Cohen et al., 1993). In the test phase the original images and variants of these were used with eyes-mouth distances compressed or extended by 4 pixels (versions -1 and $+1)$.

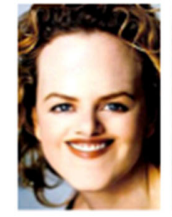

$-5$

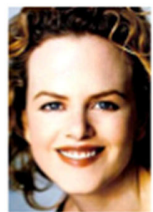

$-4$

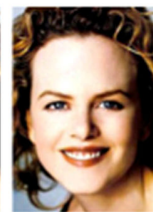

$-3$

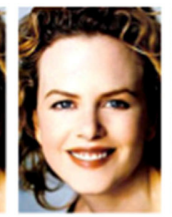

$-2$

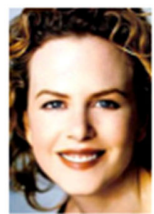

$-1$

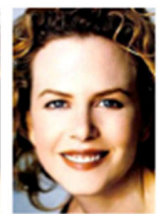

0

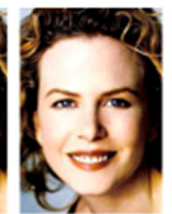

$+1$

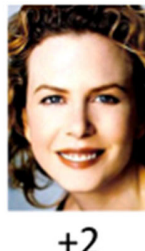

$+2$

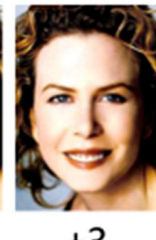

$+3$
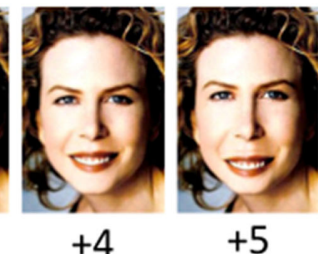

FIGURE 1 | Illustration of the stimulus continuum with the original face in the center (0), the adapting stimuli at the far ends $(-5$ and +5$)$, and the test stimuli in between $(-2$ and +2$)$. 
For all participants the adaptation phase was carried out in the formal experimental laboratory setting. In this condition the program was run on a $17^{\prime \prime}$ eMac desktop computer with completely white coating equipped with a white mouse and a white keyboard. The room was a laboratory explicitly signed as an "experimental lab" equipped with four testing cubicles, in which up to four individuals were tested in parallel. The walls, the cubicles, and the tables were also of white color; the windowless room was illuminated by artificial light of neon lamps. The experimenter gave instructions in an emphasized clear tone asking for strict obedience and an absolutely silent atmosphere. Making all these arrangements, we attempted to induce a strong laboratory atmosphere.

For the test phase, participants in the informal leisure room setting were invited to a leisure room of the department, situated in a different wing from the previously described lab, with a sofa placed at the window and a palm tree beside. The participants were invited to the room more like a guest than a participant. They were tested individually while personnel of the department were communicating with each other about different topics of social life not linked with the experiment. The test equipment consisted of a silver PowerBook 17" laptop which the participants had to place on their lap in a very informal way. A very kind person in charge, different from the experimenter in the adaptation phase, provided pieces of instructions not explicitly asking for silence, obedience, or strict conduction of the experiment.

Participants in the control group under the formal experimental laboratory setting, in contrast, found the same environmental, situational, and social properties as in the adaptation phase.

As the adaptation phase was always conducted in the formal experimental laboratory setting, but the test phase took place either in the laboratory or the leisure setting, we will term the main experimental variable episodic compatibility with the two levels laboratory (fixed settings) and ecological (variable settings).

\section{PROCEDURE}

The experiment consisted of three phases: adaptation, delay, and test.

\section{Adaptation phase}

During the adaptation phase, participants were tested in the formal experimental laboratory setting. One third of them were exposed to the strongly compressed versions $(-5)$, the original versions $(0)$, or the strongly extended versions $(+5)$ of celebrity faces, respectively. Faces assigned to the different compression sets were counterbalanced across participants. Further, participants were exposed either to image A or image B of a particular individual (see Figure 2).

The pictures assigned to the third stimulus set were not shown during adaptation. Each picture was presented 15 times during the adaptation phase fully randomized in order in five different screen positions for three different presentation durations: 2 , 3, or $4 \mathrm{~s}$. The unpredictability of presentation positions and durations aimed to exclude specific retinal effects and to ensure cognitive challenging characteristics of the task. Stimulus presentation was preceded by a sequence starting with a fixation cross $(500 \mathrm{~ms})$ in the center of the target position, followed by a rectangular frame $(200 \mathrm{~ms})$ used to guide participants' attention. After each stimulus presentation,

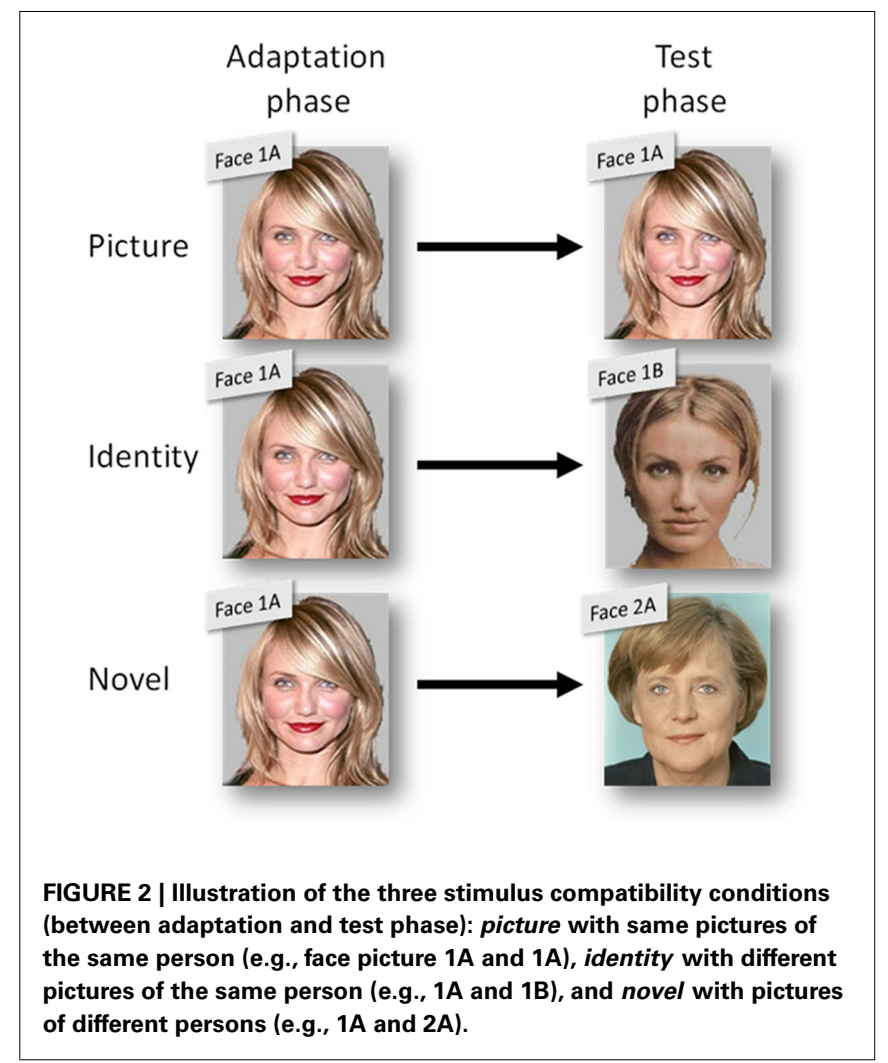

participants were requested to do a gender-decision cover task that demanded to decide what gender the stimulus belonged to as quickly as possible after it had disappeared.

\section{Delay phase}

Adaptation and test phase were separated by an interval that was at least 7 days long (mean delay: 7.1 days, range: 7-10 days). During this time, participants did not return to the lab and no specific instructions were given.

\section{Test phase}

After the delay phase, the participants had to select the veridical face out of two versions of each face (two AFC task; original vs. -2 , and original vs. +2 ). As in Carbon et al. (2007), we explicitly instructed the participants to base their decisions on their world knowledge (images known from TV/media/movies), and not on any images they had seen in the experimental context before. We also did not refer to any point of the previous adaptation phase conducted in the formal experimental laboratory setting. The participants had to reply to the question "Which is the veridical version of the face?" In each trial, either the slightly compressed version $(-2)$ or the slightly extended version $(+2)$ was presented side by side with the original one $(0)$. Each celebrity was shown four times with the various versions appearing in different, randomized locations (original left/compressed right; original right/compressed left; original left/extended right; original right/extended left).

In order to test the generalization of possible adaptation effects, the within-subject factor stimulus compatibility was manipulated on three levels: In the case of the celebrities included in the first 
stimulus set, participants were tested with exactly the same picture that had been used for adaptation (picture). For the celebrities in the second stimulus set, the alternative image of the same individual was used for testing (identity). Additionally, the test phase included one image (A or B) of each celebrity out of the third stimulus set that had not been shown during adaptation (novel).

Importantly, participants in the laboratory group were tested in the same environmental, situational, and social context as in the adaptation phase, whereas participants in the ecological group were invited in a very informal way to the informal leisure room test setting where the context was massively changed between adaptation and test phase.

\section{RESULTS}

The analyzed dependent variable was chosen target, a variable with a definition range between -1 and +1 , corresponding to constantly selecting the relatively compressed (i.e., versions -2 or 0 ) or relatively extended (i.e., versions +2 or 0 ) versions, respectively, while the value 0 means that on average participants have chosen the original face configuration. Chosen target was determined by the mean selections of each participant. Selections were scored according to their picture version: a score of -2 was used for -2 versions, 0 for 0 , and +2 for +2 ).

A three-way mixed-design analysis of variance (ANOVA) was calculated with the two between-subjects factors episodic compatibility (ecological: change of the episodic activation between adaptation and test phase from lab setting to leisure context; laboratory: no change of the episodic activation, both phases conducted in lab setting) and adaptation $(-5=$ strongly compressed, $0=$ original, $+5=$ strongly extended adaptors) and the within-subject factor stimulus compatibility (levels picture, identity, novel).

The only significant main effect we found was for adaptation, $F(2,102)=23.6, p<0.0001, \eta_{p}^{2}=0.316$, with $M_{-5}=-0.143, M_{\text {original }}=0.003$, and $M_{+5}=0.151$ (Bonferronicorrected post hoc comparisons revealed significant differences between all possible pairs, $p s<0.0001$ ). This demonstrates reliable and specific adaptation effects that were still observable after 7 days. The only further significant effect was the interaction between adaptation and stimulus compatibility, $F(4,204)=7.2$, $p<0.0001, \eta_{p}^{2}=0.124$, indicating differential amounts of adaptation for the different degrees of stimulus compatibility. Although adaptation was strongest for stimulus compatibility picture, $F(2,102)=25.6, p<0.0001, \eta_{p}^{2}=0.334$, followed by stimulus compatibility identity, $F(2,102)=19.8, p<0.0001, \eta_{p}^{2}=$ 0.279 , the simple main effect of adaptation for condition novel was still significant, $F(2,102)=6.3, p=0.0028, \eta_{p}^{2}=0.109$. Most importantly, we didn't find any effect of environmental condition (see Figure 3) - neither a main effect nor an interaction, $p s>0.298$ (see Figures 3 and 4).

Participants, who adapted to the original versions of the faces, did not show any perceptual biases in either direction.

\section{DISCUSSION}

As documented previously, incidental perception of geometrically distorted depictions of famous faces presented during a genderdecision task lead to sustained adaptation effects (Webster and Macleod, 2011). Adaptation was strongest when participants had

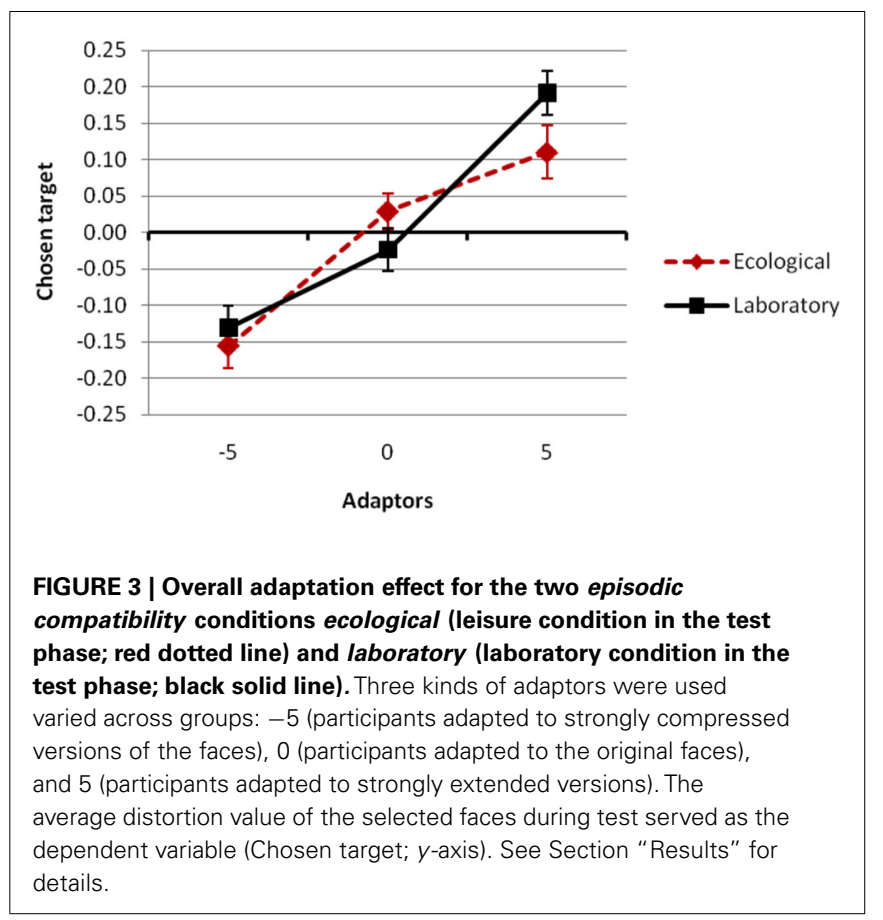

to evaluate the veridicality of celebrities' depictions that they had already seen in the adaptation phase (stimulus compatibility picture), followed by different depictions of the same celebrities (identity), and depictions of celebrities that had not yet appeared within the experimental context (novel). Thus, even the veridicality decision on faces that had not been perceived in distorted versions during the adaptation phase of the experiment was systematically biased indicating adaptation effects being in action in at least three ways: (1) picture-specific effects, demonstrated by stronger effects for condition picture compared to identity, (2) identity-specific effects, demonstrated by stronger effects for conditions picture and identity compared to novel, and (3) general adaptation effects, demonstrated by significant effects for condition novel. This finding is compatible with the results of Carbon et al. (2007) who used a different test paradigm with a 1-out-of-11 face-selection task.

The overall adaptation effects still being active after seven or more days might be an indication of effects caused by permanent shifts and tuning of the cognitive representations of faces (cf. Carbon and Ditye, 2011). Integrating new visual information in already existing representations is a core mechanism in order to recognize and discriminate effectively between face exemplars. We suggest a continuous normalization process that is in place to tune face-selective neural systems and thus maximize their sensitivity range. A similar mechanism has been shown in the domain of color-adaptation (Vul et al., 2008). Two aspects of our results are compatible with the assumption that a general tuning mechanism modifies the whole facial representation system toward recently encountered, and highly distinctive, visual information (Rhodes et al., 2003): First, adaptation effects were still existent after 1 week, and, second, we have found general adaptation effects documented by the stimulus compatibility condition 

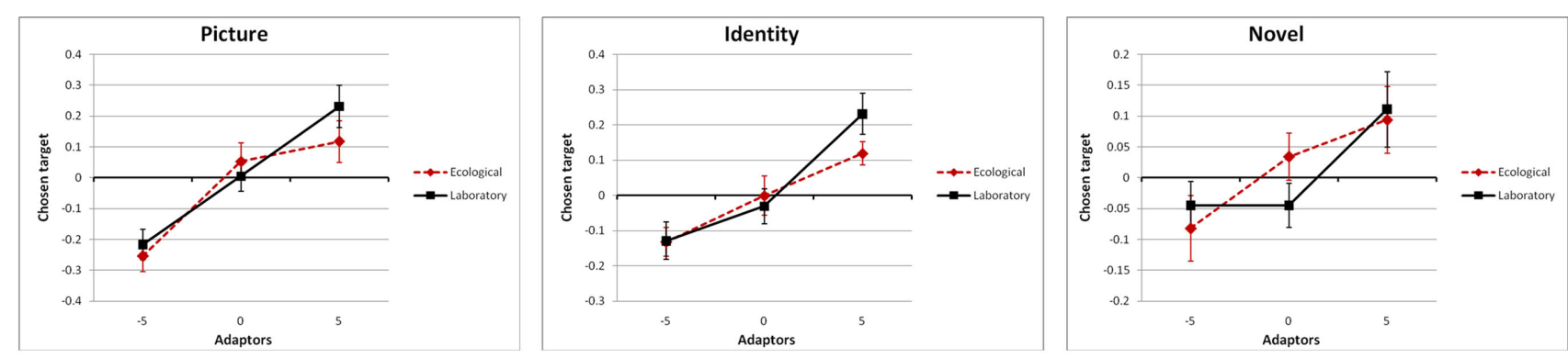

FIGURE 4 | Adaptation effects for the stimulus compatibility conditions picture (same depiction of the same persons in the adaptation and test phase), identity (different depictions of the same persons), and novel (depictions of different persons), split by the episodic compatibility conditions ecological (red dotted line) and laboratory (black solid line). novel. This interpretation is also compatible with recent findings concerning adaptation effects of aesthetic appreciation found in the domains of artworks (Carbon, 2011) and of product design, for instance for cars (Carbon, 2010) and chairs (Faerber et al., 2010).

Most importantly with respect to the aim of this paper, even 1 week after adaptation, the directed effects on face veridicality decisions were not limited to typical, highly artificial laboratory environments. They also transferred to a test setting that was very different from the adaptation setting. Our findings also support the results of a recent study showing that the size of face adaptation effects is a function of adaptation duration (Strobach et al., 2011). On these grounds we suggest to exclude simple episodic learning effects sensu Tulving's (1972) definition as the major explanation for these adaptations. Instead, we favor the idea of adaptation effects being based on the systematic tuning and deflection of the whole face space (Valentine, 2001). According to these models, every face in the face space is coded by its deviation from one or more general face norms, which are abstracted from all faces ever encountered. This view is also in line with studies on facial attractiveness (Valentine et al., 2004) and the explanation of systematic changes of aesthetic appreciation given by Faerber and Carbon (2010)

\section{ACKNOWLEDGMENTS}

The authors are indebted to Vicki Bruce's suggestion in a face symposium on the International Congress of Psychology in Berlin 2008 to ecologically test the adaptation effects we presented there. Thank you very much! Moreover, we would like to thank Vera Hesslinger for proofreading the text and Mark Mussner, Käthe Siuda, and Patrick Prüßen for conducting the experiments.

\section{REFERENCES}

Barrett, S. E., and O’Toole, A. J. (2009). Face adaptation to gender: does adaptation transfer across age categories? Vis. Cogn. 17, 700-715

Benton, C. P., Jennings, S. J., and Chatting, D. J. (2006). Viewpoint dependence in adaptation to facial identity. Vision Res. 46, 3313-3325.

Carbon, C. C. (2010). The cycle of preference: long-term dynamics of aesthetic appreciation. Acta Psychol. (Amst.) 134, 233-244.

Carbon, C. C. (2011). Cognitive mechanisms for explaining dynamics of aesthetic appreciation. i-Perception 2, 708-719.

Carbon, C. C., and Ditye, T. (2011). Sustained effects of adaptation on the perception of familiar faces. J. Exp. Psychol. Hum. Percept. Perform. 37, 615-625.

Carbon, C. C., and Leder, H. (2005). Face adaptation: changing stable representations of familiar faces within minutes? Adv. Exp. Psychol. 1, $1-7$.

Carbon, C. C., and Leder, H. (2006). The Mona Lisa effect: is "our" Lisa fame or fake? Perception 35, 411-414.
Carbon, C. C., Strobach, T., Langton, S., Harsanyi, G., Leder, H., and Kovacs, G. (2007). Adaptation effects of highly familiar faces: immediate and long lasting. Mem. Cognit. 35, 1966-1976.

Cohen, J. D., Macwhinney, B., Flatt, M., and Provost, J. (1993). PsyScope: a new graphic interactive environment for designing psychology experiments. Behav. Res. Methods Instrum. Comput. 25, 257-271.

Faerber, S. J., and Carbon, C. C. (2010). On the role of visual adaptations on aesthetic appreciation via changes of the object space. Perception 39, 76-76.

Faerber, S. J., Leder, H., Gerger, G., and Carbon, C. C. (2010). Priming semantic concepts affects the dynamics of aesthetic appreciation. Acta Psychologica 135, 191-200.

Fang, F., Ijichi, K., and He, S. (2007). Transfer of the face viewpoint aftereffect from adaptation to different and inverted faces. J. Vis. 7, 1-9.

Hills, P. J., Elward, R. L., and Lewis, M. B. (2010). Cross-modal face identity aftereffects and their relation to priming. J. Exp. Psychol. Hum. Percept. Perform. 36, 876-891.

Hole, G. (2011). Identity-specific face adaptation effects: evidence for abstractive face representations. Cognition 119, 216-228.

Kloth, N., and Schweinberger, S. R. (2008). The temporal decay of eye gaze adaptation effects. J. Vis. 8 , $1-11$.

Kovacs, G., Zimmer, M., Harza, I., Antal, A., and Vidnyanszky, Z. (2005). Position-specificity of facial adaptation. Neuroreport 16, 1945-1949.

Leopold, D. A., O'toole, A. J., Vetter, T., and Blanz, V. (2001). Prototypereferenced shape encoding revealed by high-level after effects. Nat. Neurosci. 4, 89-94.

Leopold, D. A., Rhodes, G., Muller, K. M., and Jeffery, L. (2005). The dynamics of visual adaptation to faces. Proc. R. Soc. Lond. B Biol. Sci. 272, 897-904.

Little, A. C., DeBruine, L. M., and Jones, B. C. (2005). Sex-contingent face after-effects suggest distinct neural populations code male and female faces. Proc. R. Soc. Lond. B Biol. Sci. 272, 2283-2287.
Rhodes, G., Jeffery, L., Clifford, C. W. G., and Leopold, D. A. (2007). The time course of higher-level face aftereffects. Vision Res. 47, 2291-2296.

Rhodes, G., Jeffery, L., Watson, T.-L., Clifford, C.-W. G., and Nakayama, K. (2003). Fitting the mind to the world: face adaptation and attractiveness aftereffects. Psychol. Sci. 14, 558-566.

Strobach, T., Ditye, T., and Carbon, C. C. (2011). Long-term adaptation effects of highly familiar faces: the influence of inspection length during prior testing. Perception 40, 1000-1004.

Tulving, E. (1972). "Episodic and semantic memory," in Organization of Memory, eds. E. Tulving and W. Donaldson (New York: Academic Press), 381-403.

Valentine, T. (2001). "Face-space models of face recognition," in Computational, Geometric, and Process Perspectives on Facial Cognition: Contexts and Challenges, eds. M. J. Wenger and J. T. Townsend (Hillsdale, NJ: Lawrence Erlbaum), 83-113. 
Valentine, T., Darling, S., and Donnelly, M. (2004). Why are average faces attractive? The effect of view and averageness on the attractiveness of female faces. Psychon. Bull. Rev. 11, 482-487.

Vul, E., Krizay, E., and MacLeod, D. I. A. (2008). The McCollough effect reflects permanent and transient adaptation in early visual cortex. $J$. Vis. 8, 1-12.

Webster, M. A., Kaping, D., Mizokami, Y., and Duhamel, P. (2004).
Adaptation to natural facial categories. Nature 428, 557-561.

Webster, M. A., and Macleod, D. I. A. (2011). Visual adaptation and face perception. Philos. Trans. R. Soc. Lond. B Biol. Sci. 366, 1702-1725.

Webster, M. A., and Maclin, O. H. (1999). Figural aftereffects in the perception of faces. Psychon. Bull. Rev. 6, 647-653.

Conflict of Interest Statement: The authors declare that the research was conducted in the absence of any commercial or financial relationships that could be construed as a potential conflict of interest.

Received: 25 August 2011; accepted: 04 January 2012; published online: 24 January 2012.

Citation: Carbon $\mathrm{C}-\mathrm{C}$ and Ditye $T$ (2012) Face adaptation effects show strong and long-lasting transfer from lab to more ecological contexts. Front. Psychology 3:3. doi: 10.3389/fpsyg.2012.00003

This article was submitted to Frontiers in Perception Science, a specialty of Frontiers in Psychology.

Copyright (C) 2012 Carbon and Ditye. This is an open-access article distributed under the terms of the Creative Commons Attribution Non Commercial License, which permits non-commercial use, distribution, and reproduction in other forums, provided the original authors and source are credited. 